\title{
Comprehensive Nursing Intervention for the Management of a Tip-conductive Peripherally Inserted Central Catheter in Oncology Patients
}

\author{
Yu Xia Yin ${ }^{1,2}$, Lin Ping Song ${ }^{3}$, Ming Kun Cao², Tian Heng Lu ${ }^{2}$, Hai Jun Zhang, 2, * \\ ${ }^{1}$ Department of Vascular \& Intervention, Tenth Peoples' Hospital of Tongji University, Shanghai, China \\ ${ }^{2}$ National United Engineering Laboratory for Biomedical Material Modification, Dezhou, China \\ ${ }^{3}$ The Second Department of Tumor, First Affiliated Hospital of PLA General Hospital, Beijing, China
}

Email address:

zhanghaijun@tongji.edu.cn (Hai Jun Zhang)

${ }^{*}$ Corresponding author

\section{To cite this article:}

Yu Xia Yin, Lin Ping Song, Ming Kun Cao, Tian Heng Lu, Hai Jun Zhang. Comprehensive Nursing Intervention for the Management of a Tip-conductive Peripherally Inserted Central Catheter in Oncology Patients. Cancer Research Journal. Vol. 7, No. 4, 2019 , pp. $157-160$. doi: $10.11648 /$ j.crj.20190704.16

Received: November 4, 2019; Accepted: November 22, 2019; Published: December 4, 2019

\begin{abstract}
Peripherally inserted central catheters (PICCs) play a great role in infusion of chemotherapy, parenteral nutrition, and intravenous fluid. It has the advantages of safety, convenience, long duration and low infection rate compared to central venous catheters (CVCs), becoming more and more popularized in clinical application. However, the clinical application of PICCs also causes complications, such as local infection, phlebitis, deep venous thrombosis, catheter malposition and breakage as well as liquid extravasation. The total complications rate varies mainly for three aspects: patients-related, catheter-related and operation-related. Disease types and severity of patients have been deeply researched. More concerns have been paid on the technology of catheter and operation to decrease the related complications. The intracavitary electrocardiogram (IC-ECG) method has been developed to be a safe, accurate and highly cost effective method. However, the traditional IC-ECG method is complicated and time consuming. Many researches have carried out to overcome the shortcomings of traditional methods. The tip-conductive PICC method came into being. The application of tip-conductive PICC greatly increases the first-attempt successful tip location and the tip location satisfactory. In this article, we reviewed the principle and characteristics of tip-conductive PICC, and comprehensive nursing care intervention including puncture, infection prevention, mechanical phlebitis, catheter blockage and catheter heterotopia and rupture.
\end{abstract}

Keywords: Peripherally Inserted Central Catheters (PICC), Tip-conductive, Intracavitary Electrocardiogram (IC-ECG), Comprehensive Nursing Care

\section{Introduction}

The incidence of cancer in China is increasing every year and the treatment of tumors is currently the focus of considerable medical research [1]. Surgery is often required for the treatment of tumors, and catheter insertion is needed for the patients as an adjunctive treatment. Peripherally inserted central catheter (PICC) is an intubation through a peripheral vein into central vein. Compared to the traditional subclavian venous intubation, the femoral vein intubation and the internal jugular vein intubation, PICC has advantages of small trauma, simple operation and long indwelling time and so widely used in oncology patients [2]. There is no limitation of PICC usage with respect to age, gender, type of disease. The successful PICC insertion technique has three development, the anatomical landmark method, the ultrasound technology and the intracavitary electrocardiogram (IC-ECG) method $[3,4]$. The anatomical landmark method is the basic skill for PICC placement. The ultrasound technology is well applied recently for the clinical benefits of vein assessment, venipuncture and ruling-out gross malpositions $[5,6]$. The intracavitary electrocardiogram (IC-ECG) method has been proved to be safe, accurate and highly cost effective, for it saves the expenses related to post-procedural X-ray 
confirmation and possible repositioning of malpositioned catheters [7, 8]. However, the traditional IC-ECG method acts by using a column of saline contained in the catheter as an intracavitary electrode. The operation process is complicated and time consuming. Recently, a tip-conductive PICC was designed and fabricated by adding some functional materials to the silicone to make the catheter tip conductive. The conductive tip and guide wire together act as the intracavitary electrode, so electrical signal was output on the electrocardiograph screen. Mature and effective clinical insertion is the primary condition for reducing catheter-related complications. The second is the PICC catheter itself, and another is nursing care. PICC related complications contain catheter blockage, catheter heterotopia, unplanned extubation, infection, phlebitis and thrombosis. In some reports, the incidence rates of all complications in patients at late stage were as much as $30 \%$ [9]. PICC complications may be fatal if not treated with effective and timely nursing care. Many effort has been made to decrease the complications, including promoting the catheter materials modification, improving the puncture procedure, fully vein evaluation and comprehensive nursing care et al. The application of tip-conductive PICC increases the first-attempt successful tip location from $78.9 \%$ to $91.7 \%$. Moreover, tip location satisfactory has been increased from $86.8 \%$ to $99.3 \%$ [10]. This article reviews the principle and procedure of tip-conductive PICC, and comprehensive nursing care intervention.

\section{Tip-conductive PICC}

Traditional IC-ECG method is limited for the complex procedure. It need saline flushing, so the electrical signal is not stable. Tip-conductive PICC can overcome this shorten. The conductive tip and guide wire together act as the intracavitary electrode, so the electrical signal is stable, accurate and immediate. The catheter placement is as follows: advance tip-conductive PICC gently until $5 \mathrm{~cm}$ remaining, after which IC-ECG is performed according to the standard technique. As the catheter is slowly advanced into the SVC, the P-wave gradually increased, reaching a maximal peak at the CAJ. As the catheter enters the right atrium, a diphasic $\mathrm{P}$ wave appears and the catheter should be retracted slowly to return to the position of maximal peak $\mathrm{P}$-wave, with no negative components [10]. Patients with the following cardiovascular conditions such as valve heart disease, atrial fibrillation, supraventricular tachycardia, pulmonary heart disease, pacemaker implantation, history of cardiac surgery, etc., which may affect $\mathrm{P}$-waves, should not use the tip-conductive PICC [11].

\section{Nursing Care}

Patient's illness and physical condition should be comprehensively assessed before vessel puncture. The peripheral venous state of patients should be basically checked and the vein with excellent angioplerosis and easy manipulation should be selected for puncture. Normally the basilic vein is selected for priority, followed by median cubital vein and cephalic vein [12]. Prior to the intubation the patients should be informed in health knowledge so that they comprehensively understood their body situation and the principle of treatment and strengthen the cooperation with medical personnel in treatment, and then the adverse reactions caused by their nervousness can be eliminated.

\subsection{Patients Receiving Nursing Care for Puncture}

Patients lie on side in horizontal bed, heading to one side and the skin site for intubation was strictly disinfected according to aseptic requirements of PICC catheterization. A vein puncture needle at $30^{\circ} \mathrm{C}$ was used for intubation and fixed when blood flowed backwards in the needle and was connected with a duct and a heparin cap. The whole operation is finished in strict aseptic process. Within 24 hours after the operation the puncture site was covered with sterilized gauze to prevent infection and bleeding, followed by fixation with reticulated elastic bandage [13]. At the same time the skin surrounding the puncture site was cleaned regularly to prevent postoperative bleeding and infection.

\subsection{Nursing for Infection Prevention of Patients}

The whole puncture should be performed in strict aseptic practices. After successful intubation the local drug was replaced once a day or immediately when bleeding was found, and the heparin cap and transparent adhesive replaced on time [14]. Throughout the operation, the medical staff should closely monitor the changes of patients' skin and of tissues around the puncture site. If redness, swelling, heat and pain occurred, Bactroban or other anti-inflammatory drugs should be timely administered to lesion site. And particular attention should be paid to patients with high fever. The connection of the catheter should be regularly checked to prevent infection caused by loosening or heterotopia of the catheter.

\subsection{Nursing of Patients with Mechanical Phlebitis}

Mechanical phlebitis is one of the common complications of patients in the advanced stages of PICC after catheterization. The main reason for the phlebitis included the vein damage caused by too large size or too hard material of the catheter, and that the too short distance between the puncture point and the joint caused the infection around the puncture site after intense or frequent daily movements of the patients, or that the vein for puncture was unreasonably selected. So the nursing care of patients with phlebitis included to select thick and straight vein for puncture previously, to disinfect the puncture site repeatedly, and the continuous three-day hot compress on the puncture site since the second day post catheterization, twice a day, each for 30min. Excessive limb movement should be avoided during the catheter indwelling, but moderate amount of activities such as clench fist and loose fist was planned [15]. The catheter should be repeatedly flushed using saline before and after intravenous drip. The time point, location and depth of puncture of patients were recorded as the general information, 
and pain, swelling, inflammation and medication of the patients were recorded in details to facilitate further nursing care of patients.

\subsection{Nursing of Patients During Catheter Blockage}

Among the complications of patients with PICC on late stage the catheter blockage showed the highest incidence rate. The occurrence rate of catheter blockage is proportional to the duration of catheter indwelling, i.e., the occurrence rate of catheter blockage increased with the time prolongation after catheterization. The reason is the catheter blockage and blood cell aggregation caused by improper treatment of drugs during the intravenous infusion [16]. The nursing care of patients with catheter blockage included: reasonable choice of injection drug, elimination of drug incompatibility, the order of drugs used for infusion, 20-ml physiological saline flushing pipe used for infusion of high molecular substance or viscous substances, timing wash of catheter with saline for injection of nutrient drug or blood products and addition of $5 \mathrm{ml}$ heparin to heparin cap for sealing tube. If blood was found aggregated in catheter, the coagulation should be extracted out from the tube by using a syringe, while the coagulation should not be inward injected, otherwise severe thrombosis and vascular rupture may occur.

\subsection{Nursing of Patients with Catheter Heterotopia and Rupture}

Catheter heterotopia and rupture are more common complications in patients with PICC at late stage, and is mainly caused by improper fixation of catheter, so the exposed section of the catheter should be arranged in a S-shaped figure and fixed with sterile transparent glue in the middle to ensure reliable combination of skin and the catheter. The influence of catheter heterotopia and rupture should be explained to the family members of the patient so that they are willing to intensively monitor the patient. If a catheter rupture occurred the arm should immediately stop moving to prevent catheter getting into the body, and the catheter be removed using surgical instruments [17].

\section{Conclusion}

PICC is widely used for long-term fluid infusion, chemotherapy and parenteral nutrition in clinical treatment of cancer patients, and can effectively avoid the vein injury caused by repeated intubation. Great progress has been made in modification catheter and its use as well as nursing care in recent years to meet the therapeutic needs of the patients. Tip-conductive PICC make the insertion and position of PICC more easier and accurate, thus decreasing the chances of infection and ectopic. Comprehensive nursing care intervention significantly reduced the rate of complications, reduced the occurrence of catheter accidents and increased nursing satisfaction in oncology patients receiving a PICC. The combination of tip-conductive PICC and comprehensive nursing care strategy for PICC is worthy of clinical promotion.

\section{Conflict of Interest}

All the authors do not have any possible conflicts of interest.

\section{References}

[1] Wang AY, Sherrington C, Toyama T, Gallagher M, Cass A, Hirakawa Y, Li Q, Sukkar L, Snelling P, Jardine M. Muscle strength, mobility, quality of life and falls in patients on maintenance haemodialysis: A prospective study. Nephrology (Carlton) 2016; 2: 3-5

[2] Chang HH, Chang YK, Lu CW, Huang CT, Chien CT, Hung KY, Huang KC, Hsu CC. Statins Improve Long Term Patency of Arteriovenous Fistula for Hemodialysis. Sci Rep 2016; 6: 22197.

[3] Sousa CN, Figueiredo MH, Dias VF, Teles P, Apóstolo JL. Construction and validation of a scale of assessment of self-care behaviours anticipatory to creation of arteriovenous fistula. J Clin Nurs 2015; 24: 3674-80.

[4] Dobson L, Wong D G. Development of a Successful PICC Insertion Program. Journal of Vascular Access Devices, 2001, 6 (4): 31-34.

[5] Moureau, Nancy L. Using ultrasound to guide PICC insertion. Nursing, 2003, 33 (12): 20.

[6] Nicholson J. Development of an ultrasound-guided PICC insertion service. Br J Nurs, 2013, 19 (10): 9-17.

[7] Pittiruti M, La Greca A, Scoppettuolo G. The electrocardiographic method for positioning the tip of central venous catheters. The Journal of Vascular Access, 2011, 12 (4): 280-291.

[8] Mauro P, Daniele B, Ermanno B, et al. The intracavitary ECG method for positioning the tip of central venous catheters: results of an Italian multicenter study. Journal of Vascular Access, 2012, 13 (3): 357-365.

[9] Oliver G, Jones M. ECG-based PICC tip verification system: an evaluation 5 years on. British Journal of Nursing, 2016, 25 (19): S4-S10.

[10] Yuxia Yin, Wei Gao, Xuying Li, et al. Insertion of peripherally inserted central catheters with intracavitary ECG guidance: a randomized multicenter study in China. Journal of Vascular Access. 2018.

[11] Yin Y, Wang L, Song L, Li M, Zhang H. Should We Expand the Use of Intracavitary ECG Guidance for Insertion of Peripherally Inserted Central Catheters?. Clin Surg. 2018; 3: 2208.

[12] Adib-Hajbagheri M, Molavizadeh N, Alavi NM, Abadi MH. Factors associated with complications of vascular access site in hemodialysis patients in Isfahan Aliasghar hospital. Iran J Nurs Midwifery Res 2014; 19: 208-14.

[13] Sousa CN, Apóstolo JL, Figueiredo MH, Martins MM, Dias VF. Interventions to promote self-care of people with arteriovenous fistula. J Clin Nurs 2014; 23: 1796-802.

[14] Sousa CN, Teles P, Dias VF, Apóstolo JL, Figueiredo MH, Martins MM. Physical examination of arteriovenous fistula: The influence of professional experience in the detection of complications. Hemodial Int 2014; 18: 695-9. 
[15] Preljevic VT, BH. Sandvik L. Screening for anxiety and depression in dialysis patients: Comparison of the Hospital Anxiety and Depression Scale and the Beck Depression Inventory. Journal of Psychosomatic Research 2012; 73: 327-330.
[16] Godet M, Rousseau J. Arteriovenous fistula. Rev Infirm 2012; 180: 53-4.

[17] Robson JP Jr. A review of hemodialysis vascular access devices: improving client outcomes through evidence-based practice. J Infus Nurs 2013; 36: 404-10. 